\title{
Impacto de la formación en intervención breve. Diferencias en el abordaje hospitalario del consumo de tabaco y alcohol
}

\section{Impact of a training in brief intervention. Differences in the hospital approach of tobacco and alcohol consumption}

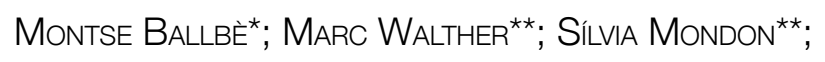

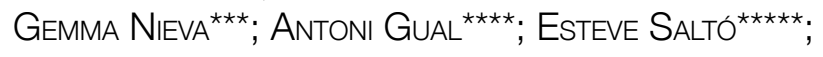
JOAN COLOM ${ }^{\star \star \star \star \star \star ~}$
*Unidad de Alcohología. Hospital Clínic de Barcelona / Institut Català d’Oncologia. Psicóloga.

**Unidad de Alcohología. Hospital Clínic de Barcelona. Médico psiquiatra.

${ }_{\star \star \star \star S}$ Servicio de Psiquiatria. Hospital Universitari Vall d'Hebron. Psicóloga.

****Unidad de Alcohología. Hospital Clínic de Barcelona. Médico psiquiatra. Jefe de la Unidad de Alcohología.

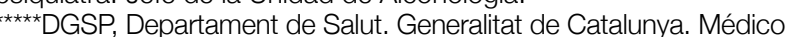
Responsable de Investigación e Información en Educación Sanitaria del Departament de Salut.

${ }^{\star \star \star \star \star \star \star}$ Generalitat de Catalunya. Médico psiquiatra. Director del Órgano Técnico de Drogodependencia.

Enviar correspondencia a:

Montse Ballbè

Unitat d'Alcohologia. Institut Clínic de Neurociències. Hospital Clínic

de Barcelona. C/ Sabino de Arana s/n, 08028 Barcelona.

E-mail: m.ballbe@gmail.com

\section{RESUMEN}

Objetivo: Evaluar el impacto de una intervención formativa para profesionales sanitarios sobre consumo de alcohol y tabaco en pacientes hospitalizados y comparar la intervención hospitalaria de dichas sustancias. Métodos: A través de dos estudios paralelos se compara, mediante cuestionarios autoadministrados, la conducta clínica declarada de 66 profesionales sanitarios frente al consumo de tabaco de sus pacientes y la intervención recibida de 169 pacientes por parte de estos profesionales, con la conducta clínica de otros 38 profesionales frente al consumo de alcohol de sus pacientes y otros 118 pacientes de diferentes unidades de hospitalización antes y después de una formación en intervención breve en tabaquismo y consumo de riesgo de alcohol respectivamente. Resultados: Se observan pocas diferencias en la conducta de los profesionales ante las dos sustancias. Los profesionales dicen prestar más ayuda en el caso del alcohol (36,4\% vs 10,9\% [p<.0001]) si bien, según la intervención recibida declarada por los pacientes, no se observan estas diferencias aunque si se les ha preguntado más por el consumo de alcohol (43,6\% vs 30,8\% [p<.05]). Después de la formación no se observan cambios significativos. Discusión: En estos dos estudios, la frecuencia de intervención de los profesionales sanitarios sobre el consumo de tabaco y alcohol es baja, tanto previa como posteriormente a la formación recibida, aunque se observa una mayor detección y ayuda en el caso del alcohol. Es necesario analizar otras barreras que frenan la aplicación de los conocimientos adquiridos.

Palabras clave: Conocimientos, actitudes y práctica en salud, rol del médico, atención de enfermería, educación, pacientes internos, consumo de bebidas alcohólicas, tabaquismo.

\section{ABSTRACT}

Objective: To evaluate the impact of a training intervention for health care professionals on inpatients' smoking and alcohol consumption and to compare the hospital intervention for the two substances. Methods: Through two parallel studies, we compared, using auto-administered questionnaires, the referred clinical behaviour on smoking of 66 health care professionals and the intervention received by 169 patients, with the clinical behaviour on hazardous drinking of other 38 professionals and other 118 patients from different hospital wards before and after attending a training in brief intervention on smoking cessation and hazardous alcohol drinking respectively. Results: We only found slight differences between alcohol and tobacco concerning professionals' behaviour. Professionals declared to assist more often patients with alcohol risk consumption than smoking (36,4\% vs 10,9\% [p<.0001]) although with patients those differences haven't been confirmed. They just told to have been asked more often about alcohol consumption than about smoking (43,6\% vs 30,8\% $[p<.05])$. There were no differences after the training sessions. Discussion: Before and after training, health professionals hardly intervene in patients with alcohol or tobacco consumption even we observed higher detection and assist for alcohol risk consumption. It is necessary to examine other barriers to the application of the knowledge gained.

Key words: Health knowledge, attitudes, practice, physician's role, nursing care, education, inpatients, alcohol drinking, smoking. 


\section{INTRODUCCIÓN}

E tabaco y el alcohol constituyen las dos principales causas de muerte evitable según el informe de la OMS del año $2002^{1}$ y los costes sanitarios asociados a su consumo o abuso son muy altos ${ }^{2,3}$. Pese a ello, la detección y manejo del consumo o abuso de estas sustancias en el paciente por parte de los profesionales sanitarios es poco frecuente. No obstante, en el ámbito de la atención primaria la detección y manejo del abuso y consumo del alcohol y el tabaco respectivamente es más frecuente que en el ámbito hospitalario. Asi, Aalto et $\mathrm{al}^{4}$ (2001) observaron que la mayoría de los médicos de atención primaria consideraban como una parte normal de su trabajo el detectar y aconsejar a sus pacientes con consumo de riesgo de alcohol. También en atención primaria, Cerrada et $\mathrm{al}^{5}$ (2005) constataron que la mayoría de los profesionales de la salud preguntaban y aconsejaban a sus pacientes sobre el cese tabáquico.

En cuanto al ámbito hospitalario, la frecuencia de intervención de los profesionales sanitarios sobre pacientes fumadores y bebedores de riesgo es todavía insuficiente ${ }^{6,7,8}$ y aún es menor cuando los pacientes no presentan patologías relacionadas con el consumo de estas sustancias quedando en un segundo término las intervenciones preventivas ${ }^{9}$.

Las principales barreras que los profesionales de la salud aluden como razones de su baja intervención son la poca formación, la falta de tiempo percibida, el no considerarlo su área de responsabilidad y la falta de motivación, entre otras. Se ha apuntado la falta de formación como el motivo que explica en mayor medida la poca intervención ${ }^{4}, 10$. Pese a ello, son escasos los estudios realizados sobre el impacto de programas de formación para profesionales en este tema aunque existen tres estudios en el ámbito de la atención primaria que muestran resultados positivos ${ }^{11}, 12,13$

El alcohol y el tabaco son las dos sustancias adictivas legales más importantes en España y en el mundo occidental en general, sin embargo, existen algunas diferencias en su historia en cuanto a su percepción médica y social. Así, en el ámbito médico existe conciencia desde hace mucho tiempo del potencial adictivo del alcohol pero bastante menos del tabaco. Paralelamente, el cambio de actitud de la sociedad hacia el consumo del tabaco y el desarrollo de técnicas de detección y de intervención en tabaquismo son muy recientes. Por otro lado, el concepto de "consumo de riesgo" del alcohol también es nuevo, aunque se conoce la sustancia desde hace miles de años por su capacidad adictiva.

Dos estudios realizados fuera de España han comparado algunos aspectos de la intervención sobre estas dos sustancias, encontrando diferencias en la detección e intervención del tabaquismo y del consumo de riesgo de alcohol por parte de los profesionales sanitarios. En atención primaria, Aira y colaboradores ${ }^{14}$ (2004) observaron que los profesionales sanitarios mencionaban con más frecuencia el consumo de tabaco que de alcohol en las historias clínicas de sus pacientes, y por otra parte, declaraban sentirse más cómodos con el abordaje preventivo del tabaquismo que del alcohol. En el ámbito hospitalario, Shourie et al. ${ }^{15}$ (2007) constataron que los profesionales sanitarios valoraban el tabaquismo de forma más consistente que el consumo de alcohol.

El objetivo de este estudio consiste en evaluar, a través de dos estudios paralelos ${ }^{16,17}$, el impacto de dos sesiones para profesionales sanitarios de un hospital general basadas en la intervención breve sistematizada en cinco elementos ("las $5 \mathrm{~A}^{\prime \prime}$ ) recomendada por la $0 \mathrm{MS}^{18}$ para el abordaje del consumo de tabaco y consumo de riesgo de alcohol y comparar la conducta clínica (abordaje) de los profesionales frente al consumo de las dos sustancias en sus pacientes.

\section{MÉTODOS}

Los dos estudios paralelos sobre tabaco y consumo de riesgo de alcohol se realizaron en 9 unidades de hospitalización del Hospital Clínic de Barcelona.

\section{Sujetos}

El grupo de profesionales evaluados fueron todos aquellos que acudieron a las sesiones de formación de cada unidad y los pacientes de esas mismas salas con el único criterio de exclusión de deterioro de la capacidad cognitiva.

\section{Estudio sobre formación en intervención en cesación tabáquica:}

a) 66 profesionales sanitarios (médicos, enfermeros/as y otros) de 5 unidades hospitalarias (psiquiatría, medicina interna, traumatología, ginecología y obstetricia). La muestra para la evaluación de los mismos profesionales después de la formación se redujo a 59 debido a la pérdida de la información de 7 profesionales. La edad media fue de 38,63 años $(d s=11,11)$ y el $84,4 \%$ eran mujeres. El $48,5 \%$ eran enfermeros/as, el 33,3\% médicos y el resto, otros profesionales sanitarios.

b) 170 pacientes consecutivos ingresados en las mismas 5 unidades para la evaluación antes de la formación y otros 170 para la evaluación después de la formación. La edad media fue de $50,84(d s=20,78$ ) y el $73,5 \%$ eran mujeres.

\section{Estudio sobre formación en intervención en consumo de riesgo de alcohol:}

a) 38 profesionales sanitarios (médicos, enfermeros/as y otros) de 4 unidades hospitalarias (psiquiatría, medicina interna, cardiología y neumología). La muestra para la evaluación de los mismos profesionales después de la formación se redujo a 34 debido a la pérdida de la información de 4 profesionales. La edad media fue de 37,65 años $(d s=11,08)$ y el 78,9\% eran mujeres. El 50\% eran enfermeros/as, el 36,8\% médicos y el resto, otros profesionales sanitarios. 
b) 118 pacientes consecutivos ingresados en las mismas 4 salas para la evaluación antes de la formación y otros 122 para la evaluación después de la formación. La edad media fue de $64,05(d s=18,81)$ y el $41,3 \%$ eran mujeres.

En cuanto a la conducta de los propios profesionales frente a las dos sustancias, una tercera parte $(35,4 \%)$ de los profesionales del estudio sobre tabaco refirió fumar, un $24,6 \%$ era ex-fumador y un $40 \%$ no fumador. En cuanto al estudio del alcohol, el $71,5 \%$ de los profesionales era bebedor de bajo riesgo, un $20 \%$ se consideraba abstemio y un $8,5 \%$ bebedor de riesgo.

\section{Instrumentos}

Para los dos estudios se evaluó mediante cuestionarios semi-estandarizados y autoadministrados:

a) Cuestionario para profesionales: Los elementos o etapas de la intervención breve realizados habitualmente y declarados por los profesionales sanitarios de las unidades mediante preguntas de respuesta categórica para cada uno de los cinco elementos de la intervención de las $5 \mathrm{~A}$ (ej.: ¿Con qué frecuencia pregunta a sus pacientes sobre el consumo de alcohol? siempre / frecuentemente / a veces / raramente / nunca). Además se preguntaba por los conocimientos farmacológicos y psicológicos percibidos, así como su propio consumo autodeclarado y actitudes frente a la sustancia (tabaco o alcohol según el estudio).

b) Cuestionario para pacientes: El consumo de dichas sustancias en pacientes ingresados en las diferentes unidades hospitalarias y los elementos de intervención breve recibidos ( $5 \mathrm{~A}$ ) según los mismos pacientes mediante ítems de respuesta dicotómica (ej.: ¿Durante este ingreso hospitalario algún profesional sanitario le ha hecho alguna pregunta sobre el consumo de bebidas alcohólicas? si / no).

La evaluación de pacientes y profesionales se realizó inmediatamente antes y 4 semanas después de la sesión de formación y fue realizada por un médico psiquiatra y una psicóloga especializados en intervención en alcohol y tabaco. Los cuestionarios para los pacientes se administraron el día de su alta hospitalaria y el proceso duró tres meses.

\section{Sesión de formación}

La intervención formativa fue de una hora de duración sobre el tabaco o el alcohol según el estudio y se impartieron los siguientes temas: a) epidemiología, b) importancia de la intervención en pacientes hospitalizados, c) intervenciones eficaces, d) detección e intervención breve sistematizada en cinco etapas (las " $5 A^{\prime \prime}$ ) recomendada por la $0 \mathrm{MS}^{18}$ : 1) Ask: averiguar si el paciente fuma o bebe en exceso, 2) Advice: aconsejar firmemente dejar de fumar o beber en exceso, 3) Assess: Apreciar (o evaluar) la disposición para dejar de fumar o beber en exceso, 4) Assist: ayudar en el abandono y
5) Arrange: acordar y programar el seguimiento, y e) tratamiento farmacológico y estrategias psicológicas.

La sesión tuvo lugar en un horario accesible al turno de trabajo de mañana y tarde (al mediodía). Se realizó una sesión de formación igual para cada unidad hospitalaria incluida en el estudio y el lugar de la sesión fue en salas ubicadas en la misma unidad. Se entregaron diplomas acreditados por la Comisión de Formación Continuada del Sistema Nacional de Salud y el Consell Català de Formació Mèdica Continuada a los asistentes. Se entregó, también, material escrito de recordatorio (folletos) sobre cómo realizar la intervención breve.

\section{Análisis de datos}

El análisis estadístico de los datos obtenidos de los cuestionarios se realizó mediante el paquete estadístico SPSS (versión 12.0). Para las variables cuantitativas se utilizaron pruebas T. Para las cualitativas, se realizaron pruebas de Chi cuadrado, con corrección por continuidad de Yates o pruebas exactas de Fisher en el caso de tener frecuencias o muestras reducidas. Para la comparación de la conducta clínica de los profesionales se llevaron a cabo pruebas de Mann-Whitney para la comparación de variables nominales con ordinales.

\section{RESULTADOS}

Las muestras de profesionales de los dos estudios no se diferenciaron estadísticamente en cuanto al sexo, la edad o la profesión.

No se observaron diferencias significativas en el nivel de conocimientos de los profesionales sobre herramientas terapéuticas farmacológicas y psicológicas entre las dos sustancias. Así, antes de la formación un $25,8 \%$ y un $25 \%$ de los profesionales declaraba tener "suficientes o muchos conocimientos" sobre intervenciones psicológicas para el abordaje del tabaco y del alcohol respectivamente y un $33,3 \%$ declaraba poseer "suficientes o muchos conocimientos"sobre intervenciones farmacológicas para ambas sustancias (el resto tenia pocos o ningún conocimiento). Después de la formación, los profesionales percibieron que sus conocimientos habían aumentado significativamente para intervenir en el consumo de tabaco (un 23,4\% más para las intervenciones psicológicas y un 27,1\% más para las farmacológicas) aunque no hubo cambios significativos para el alcohol ${ }^{16,17}$. Pese a ello, en el caso del alcohol, después de la formación los profesionales conocían mejor los límites de consumo de alcohol para hombres y mujeres según la OMS (de un 7,8\% que lo sabía a un $29,0 \%$ después de la formación; $p<.05$ ) y la correspondencia de gramos de alcohol por unidad de bebida estándar (de un $34,2 \%$ que lo sabía a un $77,4 \%$ después de la formación; $p<.001)$. 
Respecto a la intervención declarada por los profesionales sanitarios sobre el tabaco y el alcohol en sus pacientes, previa a la formación (ver tabla 1), no se observaron diferencias significativas en el porcentaje en que se preguntaba por el consumo de dichas sustancias ni en el grado en el que se aconsejaba abandonar o moderar el consumo de las mismas. Por otro lado, los profesionales evaluaban con mayor frecuencia el consumo de alcohol que el de tabaco cuando no existía patología relacionada con la sustancia (37,5\% para el alcohol vs 17,2\% para el tabaco $[p<.0001])$. Existían también diferencias significativas en el grado en que los profesionales decian ayudar a sus pacientes a cesar o moderar el consumo de estas sustancias siendo mucho mayor su actuación para el alcohol. Así, antes de la formación, un 57,1\% de los profesionales refería ayudar a sus pacientes a controlar su consumo de alcohol siempre o frecuentemente frente a un $24,6 \%$ para el tabaco en los pacientes con enfermedades relacionadas con dichas sustancias $(p<.0001)$. La misma tendencia se observaba cuando los pacientes no presentaban enfermedades relacionadas con la sustancia $(36,4 \%$ para el alcohol frente a un $10,9 \%$ para el tabaco $[p<.0001])$. Finalmente, no hubo diferencias significativas en cuanto al porcentaje en que los profesionales decian registrar "siempre o frecuentemente" el consumo de estas sustancias en las historias clínicas de sus pacientes. Se invalidó el análisis del componente acordar seguimiento por un error en el cuestionario.

Tabla 1. Comparación de la conducta de los profesionales frente a las dos sustancias (intervención $5 \mathrm{~A}$ ) en porcentajes.

\begin{tabular}{|c|c|c|c|c|}
\hline \multicolumn{2}{|c|}{$\begin{array}{l}\text { Conducta clinica } \\
\text { (antes de la formación) }\end{array}$} & \multirow{2}{*}{$\begin{array}{c}\begin{array}{c}\text { Tabaco } \\
(\mathrm{N}=66)\end{array} \\
70,3 \%\end{array}$} & \multirow{2}{*}{$\begin{array}{c}\begin{array}{c}\text { Alcohol } \\
(\mathrm{N}=38)\end{array} \\
52,6 \%\end{array}$} & \multirow{2}{*}{$\begin{array}{c}U ; p^{+} \\
U=1039,5 \\
p=0,194\end{array}$} \\
\hline & Con patologia & & & \\
\hline (Averiguar) & Sin patología & $39,7 \%$ & $47,2 \%$ & $\begin{array}{c}U=1044,0 \\
p=0,501\end{array}$ \\
\hline \multirow{2}{*}{$\begin{array}{l}\text { ADVICE } \\
\text { (Aconsejar) }\end{array}$} & Con patologia & $75,4 \%$ & $63,2 \%$ & $\begin{array}{c}U=1229,5 \\
p=0,967\end{array}$ \\
\hline & Sin patologia & $31,1 \%$ & $45,7 \%$ & $\begin{array}{l}U=913,5 \\
p=0,122\end{array}$ \\
\hline \multirow{2}{*}{$\begin{array}{l}\text { ASSESS } \\
\text { (Apreciar / Evaluar) }\end{array}$} & Con patología & $43,1 \%$ & $57,1 \%$ & $\begin{array}{l}U=907,5 \\
p=0,088\end{array}$ \\
\hline & Sin patologia & $17,2 \%$ & $37,5 \%$ & $\begin{array}{l}U=583,0 \\
p=0,000\end{array}$ \\
\hline \multirow{2}{*}{$\begin{array}{l}\text { ASSIST } \\
\text { (Ayudar) }\end{array}$} & Con patologia & $24,6 \%$ & $57,1 \%$ & $\begin{array}{l}U=640,5 \\
p=0,000\end{array}$ \\
\hline & Sin patologia & $10,9 \%$ & $36,4 \%$ & $\begin{array}{l}U=517,5 \\
p=0,000\end{array}$ \\
\hline \multirow{2}{*}{ REGISTRAR } & Con patología & $53,1 \%$ & $54,1 \%$ & $\begin{array}{l}\mathrm{U}=1179,0 \\
\mathrm{p}=0,971\end{array}$ \\
\hline & Sin patologia & $38,1 \%$ & $36,1 \%$ & $\begin{array}{c}U=1089,0 \\
p=0,738\end{array}$ \\
\hline
\end{tabular}

$\%$ de los profesionales que contestaron realizar la intervención siempre o frecuentemente (\% restante contestaron realizar la intervención a veces, raramente o nunca). Con patologia: pacientes con patologia relacionada con la sustancia. Sin patologia: el resto de los pacientes.

${ }^{+} \mathrm{U}=$ Prueba de Mann-Whitney; $p=$ significación.

No se encontraron diferencias significativas en el porcentaje de intervención en alcohol y tabaco de los profesionales según si eran o no fumadores o consumidores de riesgo de alcohol.
En cuanto a la conducta clínica de los profesionales, después de la formación, tampoco se observaron cambios significativos en los resultados.

Para el estudio del tabaco, el 54,1\% de los profesionales de las unidades estudiadas acudió a la formación; el 83,3\% de los médicos y el $47 \%$ de las enfermeras/os. Para el estudio del alcohol acudieron el 57,1\% del total de profesionales de la unidades estudiadas (el 51,35\% de los médicos y el 61,7\% de las enfermeras/os).

En la muestra de pacientes, se observaron algunas diferencias entre los dos grupos de alcohol y tabaco en cuanto al sexo (el 38,1\% para la muestra de alcohol y el $77,1 \%$ para la de tabaco eran mujeres $[p<.0001])$ y en cuanto a la edad $(\bar{\chi}=66,11$ años, ds=17,7 para la muestra de alcohol y $\bar{\chi}$ $=51,54, d s=20,5$ para la muestra de tabaco; $p<.0001)$. No se observaron diferencias significativas en la muestra antes y después de la formación. Analizando la intervención recibida, antes de la sesión de formación (ver tabla 2), se constataron diferencias significativas en el porcentaje de pacientes que decian que se les había preguntado si consumían tabaco 0 alcohol. Así se les preguntó más por el consumo de alcohol que de tabaco (43,6\% para el alcohol vs 30,8\% para el tabaco; $p<.05)$, sin diferencias según la edad y el sexo del paciente. También se observaron diferencias estadísticamente significativas en el grado en que los profesionales anotaron si existía consumo en el informe de alta de manera más frecuente para el caso del tabaco $(72,9 \%$ para el tabaco vs $56,0 \%$ para el alcohol; $p<.01$ ) sin diferencias según la edad aunque sí según el sexo del paciente. Así, se anotaba con más frecuencia si existía o no consumo de tabaco en el caso del las mujeres (se anotaba el $81,4 \%$ de las veces en las mujeres y el $18,5 \%$ en los hombres; $p<.05$ ) (en especial por la inclusión de la unidad de obstetricia en la muestra del estudio de tabaco), aunque no ocurría lo mismo para el alcohol.

Tabla 2. Comparación de la intervención breve recibida según los pacientes.

\begin{tabular}{|l|c|c|c|}
\hline Intervención recibida & Tabaco & Alcohol & $p$ \\
\hline $\begin{array}{l}\text { ASK* } \\
\text { (Averiguar) }\end{array}$ & $30,8 \%$ & $43,6 \%$ & $\begin{array}{c}X^{2}=4,931 ; \\
p=0,026^{+}\end{array}$ \\
\hline $\begin{array}{l}\text { ADVICE* } \\
\text { (Aconsejar) }\end{array}$ & $21,9 \%$ & $50,0 \%$ & $\begin{array}{c}X^{2}=3,639 ; \\
p=0,056^{+}\end{array}$ \\
\hline $\begin{array}{l}\text { ASSESS** } \\
\text { (Apreciar / Evaluar) }\end{array}$ & $25,0 \%$ & $28,6 \%$ & $1,000^{*}$ \\
\hline $\begin{array}{l}\text { ASSIST* } \\
\text { (Ayudar) }\end{array}$ & $6,3 \%$ & $4,5 \%$ & $1,000^{+}$ \\
\hline $\begin{array}{l}\text { ARRANGE* } \\
\text { (Acordar seguimiento) }\end{array}$ & $3,1 \%$ & $13,6 \%$ & $0,217^{+}$ \\
\hline Registro en el informe de alta & $72,9 \%$ & $56,0 \%$ & $\begin{array}{c}X^{2}=9,257 ; \\
p=0,002^{+}\end{array}$ \\
\hline
\end{tabular}

$\%$ de pacientes que contestaron sí haber recibido la intervención (variable dicotómica SI-NO).

*Muestra total de pacientes: Tabaco $n=169$; Alcohol $n=118$

**Muestra de pacientes fumadores o bebedores de riesgo: Tabaco $n=32 ;$ Alcohol $n=14$

†Prueba de Chi cuadrado; $¥$ Chi cuadrado con corrección por continuidad de Yates; キPrueba exacta de Fisher.

En el estudio de alcohol, después de la formación los profesionales registraron con más frecuencia el consumo de 
dicha sustancia (de 56,0\% antes de la formación a 69,2\% después de la misma $[p<.05])$. Sin embargo, no se observaron diferencias significativas en la conducta clínica ante las dos sustancias para el resto de componentes de la intervención breve (aconsejar, evaluar, ayudar y acordar seguimiento) ni según la edad y el sexo del paciente. No obstante, se percibió una tendencia no significativa a aconsejar más para el consumo de alcohol (50,0\% para el alcohol vs 21,9\% para el tabaco; $p=0,056)$. La muestra para los componentes Ayudar y Acordar seguimiento se redujo debido a que no podian contestarse las preguntas si los pacientes no habian recibido el paso anterior en la intervención breve, es decir, Evaluar la disposición para dejar de fumar para poder Ayudar o Ayudar para poder Acordar seguimiento.

\section{DISCUSIÓN}

Los resultados de este estudio difieren de los de Aira ${ }^{14}$ et al. y Shourie ${ }^{15}$ et al., en los que se coincide en una mayor detección del tabaquismo que del consumo de alcohol y una mayor comodidad en el abordaje preventivo del tabaquismo quizás por realizarse en países con una conciencia de los riesgos del tabaquismo más antigua (Finlandia y Australia). Por ejemplo, en Finlandia, ya en el año 1995 se aprobó una restrictiva ley de control del tabaquismo mientras que en España ésta no se implantó hasta el año 2006.

Aunque la mayoría de profesionales pregunta por el consumo en pacientes con patología relacionada con la sustancia, finalmente sólo un 10 o 20 por ciento dice realizar toda la intervención breve. No obstante, en pacientes sin patología relacionada, sólo el 30 o 40 por ciento de los profesionales indica una intención inicial de detección (preguntar si existe consumo). Globalmente, la intervención en pacientes sin patología relacionada con las dos sustancias es muy poco frecuente, descuidándose de esta manera la vertiente preventiva de la actuación sanitaria. En general, la conducta y los conocimientos de los profesionales no presentan muchas diferencias según la sustancia (tabaco o alcohol) aunque se observa una mayor detección y ayuda en el caso del alcohol. La formación parece haber dado algunas nociones básicas en el caso del tabaquismo, generando una sensación satisfactoria de haber mejorado los conocimientos. En cambio, el comportamiento de los profesionales no se modifica significativamente. En el caso del alcohol, los profesionales no tuvieron la misma impresión de haber aprendido. En este punto, el análisis más exhaustivo de todos los resultados del estudio ${ }^{16}$ nos indicó, que sus conocimientos objetivos aumentaron. Por otra parte, el hecho de que los conocimientos farmacológicos fueran mayores en el caso del tabaco era esperable, puesto que en este estudio considerábamos el consumo de riesgo de alcohol y no la dependencia y, en este caso, la intervención farmacológica no suele ser tan importante.
Aún no constituyendo un objetivo del presente estudio, observamos que las cifras obtenidas de profesionales fumadores y bebedores de riesgo de nuestra muestra se corresponden con los porcentajes de la población general en Cataluña $\left(29,3 \%{ }^{19}\right.$, fumadores/as diarios y ocasionales), $\left(9,5 \%{ }^{20}\right.$, bebedores de riesgo), por lo que pertenecer a una profesión con un importante rol modélico en la promoción de la salud no parece influir sobre la propia actitud del profesional frente a estas dos sustancias.

Finalmente, se observa una situación similar tanto en el nivel de conocimientos como en la baja intervención de los profesionales para las dos sustancias. El contenido de ambas sesiones de formación también resulta parecido a excepción de los conceptos específicos para cada una, lo que podría dar viabilidad a sesiones de formación conjunta de coste más reducido. No obstante, es necesaria una amplia participación de los profesionales que trabajan en las unidades de ingreso hospitalario y buscar nuevas estrategias formativas más efectivas. En nuestro estudio, una sola sesión de una hora aporta conocimientos pero no cambios en la conducta del profesional sanitario. En comparación, el estudio de Unrod et al. (2007) en el ámbito de la Atención Primaria un entrenamiento individual de 40 minutos basado también en la intervención de las $5 \mathrm{~A}$ llevó a un aumento de la intervención. Por los resultados obtenidos en nuestro estudio, podría ser necesario llevar a cabo sesiones de seguimiento posteriores haciendo hincapié en un tipo de formación más práctica y abordando los problemas y barreras que encuentran los profesionales para llevar a cabo la intervención en el ámbito hospitalario mediante una búsqueda conjunta de soluciones.

En general, los datos muestran unas tasas de intervención moderadamente bajas para el consumo de tabaco y alcohol, lo que pone en evidencia la necesidad de seguir con estrategias de formación y sensibilización más efectivas y de aumentar, especialmente, el abordaje preventivo de dichas sustancias.

\section{AGRADECIMIENTOS}

Este estudio ha sido realizado y financiado parcialmente en el marco del convenio de colaboración entre el Instituto Catalán de Oncología (ICO) y la DG de Salut Pública del Departament de Salut para el desarrollo de la Red de Hospitales Sin Humo de Cataluña y a través de la Subdirección General de Drogodependencias de la DG de Salut Pública en el marco del programa "Beveu Menys".

Agradecemos la colaboración del personal sanitario de las unidades de hospitalización citadas del Hospital Clínic de Barcelona. 


\section{REFERENCIAS}

$1 \quad$ OMS. The World Health Report 2002. Reducing risks, promoting healthy life. Geneva: World Health Organization; 2002.

2 Hurley SF. Hospitalization and costs attributable to tobacco smoking in Australia: 2001-2002. Med J Aust 2006; 184: 45.

3 PHEPA, Primary Health Care European Project on Alcohol. Disponible en: http://www.gencat.net/salut/phepa/units/phepa/ html/en/dir353/index.html [última modificación el 3-5-2005; consultado el 22-3-2007].

4 Aalto M, Pekuri P, Seppa K. Implementation of brief alcohol intervention in primary care: do nurses' and general practitioners' attitudes, skills and knowledge change?. Drug Alcohol Rev 2005; 24: 555-8.

5 Cerrada Cerrada E, López Olmeda C, Bouzas Senande E, Gómez Rodríguez B, Sanz Cuesta T. Opiniones, prácticas, barreras y predisposición al cambio a la hora de dar consejo para dejar de fumar. Aten Primaria 2005; 15: 434-41.

6 Hearne R, Connolly A, Sheehan J. Alcohol abuse: prevalence and detection in a general hospital. J R Soc Med 2002; 95: 84-7.

7 Neighbor WE, Stoop DH, Ellsworth A. Smoking cessation counseling among hospitalized smokers. Am J Prev Med 1994; 10: $140-4$

8 Nieva G, Gual A, Mondon S, Walther M, Saltó E. Evaluación de la intervención mínima en tabaquismo en el ámbito hospitalario. Med Clin (Barc) 2007; 128: 730-2.

9 Frank E, Winkleby MA, Altman DG, Rockhill B, Fortmann SP. Predictors of physicians's smoking cessation advice. JAMA 1991; 266: 3139-44.

10 Twardella $\mathrm{D}$, Brenner $\mathrm{H}$. Lack of training as a central barrier to the promotion of smoking cessation: a survey among general practitioners in Germany. Eur J Public Health 2005; 15: 140-145.
11 Babor TF, Higgins-Biddle JC, Higgins PS, Gassman RA, Gould BE. Training medical providers to conduct alcohol screening and brief interventions; Subst Abus 2004; 24: 17-26.

12 Peltzer K, Seoka P, Babor T, Obot I. Training primary care nurses to conduct alcohol screening and brief interventions in South Africa. Curationis 2006; 29: 16-21.

13 Unrod M, Smith M, Spring B, DePue J, Redd W, Winkel G. Randomized controlled trial of a computer-based, tailored intervention to increase smoking cessation counseling by primary care physicians. J Gen Intern Med 2007; 22: 478-84.

14 Aira M, Kauhanen J, Larivaara P, Rautio P. Differences in brief interventions on excessive drinking and smoking by primary care physicians: qualitative study. Prev Med 2004, 38: 473-8.

15 Shourie S, Conigrave KM, Proude EM, Haber PS. Detection of and intervention for excessive alcohol and tobacco use among adult hospital in-patients. Drug Alcohol Rev 2007, 26: 127-33.

16 Walther M, Ballbè M, Mondon S, Nieva G, Gual A. Teaching hospital staff about hazardous drinking: the effect of a single intervention. Alcohol Alcohol 2008; 43: 51-2.

17 Ballbè M, Mondon S, Nieva G, Walther M, Saltó E, Gual A. Evaluación de un programa de formación de profesionales sanitarios sobre abordaje del tabaquismo en pacientes hospitalizados. Adicciones 2008; 20: 125-9.

18 OMS. Evidence based core recommendations for health care systems in Europe. Partnership Project to Reduce Tobacco Dependence. February; 2001.

19 Departament de Salut, Generalitat de Catalunya. Enquesta de Salut de Catalunya 2006. Disponible en: http://www.gencat.net/ salut/depsan/units/sanitat/pdf/r15.pdf

20 Departament de Salut, Generalitat de Catalunya. Enquesta Domiciliària sobre Consum de Drogues, 2003. Delegació del Govern per al PND. Datos para Catalunya facilitadas por la OTD. 